\title{
Performance evaluation of space time trellis coded MIMO for mobile communications
}

\author{
Gebrehiwet Gebrekrstos Lema ${ }^{1}$, Teklehaymanot Baweke Reda ${ }^{2}$, Dawit Hadush Hailu ${ }^{3}$, Tole Sutikno ${ }^{4}$ \\ ${ }^{1,2,3}$ Ethiopian Institute of Technology - Mekelle, School of Electrical and Computer Engineering, \\ Mekelle University, Ethiopia \\ ${ }^{4}$ Department of Electrical Engineering, Universitas Ahmad Dahlan, Indonesia
}

\begin{tabular}{l} 
Article Info \\
\hline Article history: \\
Received Jan 9, 2019 \\
Revised Apr 20, 2019 \\
Accepted May 3, 2019 \\
\hline
\end{tabular}

\section{Keywords:}

Combining techniques

Multipath fading

Space diversity

\begin{abstract}
The major enemy of wireless communication is multipath fading that highly degrades the received signal. Spatial diversity highly reduces deep fades of the wireless communication. Higher signal to noise ratio (SNR) requirement can be reduced by using space time coding. In this paper, the error performance of un-coded MIMO, different diversity combing methods, space time block codes and space time trellis codes are analyzed using different parameters including number of antennas, M-array modulations, trace criteria, rank and determinant. The simulation results have shown that the Bit Error Rate (BER) significantly reduces with increasing number of receiver antennas. However, the number of antennas that a mobile device can have is limited by its size and this is inconvenient for mobile communication. Hence, this paper suggests space time codes to mitigate multipath problems in mobile communication. So, BER performance of space time block codes scheme was evaluated by varying the number of transmitter and receiver antennas along with varying M-array PSK modulation orders. The results have shown that better BER is possible by integrating space time codes with spatial diversity. Finally, the error performance of space time trellis codes was evaluated by using trace, rank and determinant, and the simulation results depicted that better error performance is achieved using the proposed multipath reduction method.
\end{abstract}

Copyright $(2019$ Institute of Advanced Engineering and Science. All rights reserved.

\section{Corresponding Author:}

Gebrehiwet Gebrekrstos Lema,

Department of Electrical and Computer Engineering,

Mekelle University,

Mekelle, Tigray, Ethiopia.

Email: g.jcool.com@gmail.com

\section{INTRODUCTION}

Multipath fading has been a big challenge for communication engineers. In the transmission of a single frequency signal using single transmitter antenna, the signal follows different paths to arrive at the receiver where the paths followed can have different phase, amplitude and delays which when combined can produce destructive interference or constructive interference. Mobile communications are subject to multipath fading and the common reasons includes when the mobile user is moving. The mobile station or user is likely to be moving, and as a result the path lengths of all the signals being received are changing. Moreover, automobiles and people can cause reflections that results in to significant effect on the received signal. Accordingly, multipath fading has a major problem on cellular telecommunications. Deeply faded signals are hard to detect and process.

Diversity techniques are used to overcome the multipath fading. Many diversity techniques exist to prevent deep fading such as time diversity where a symbol is transmitted at different time intervals hoping that the wireless environment the device experiences is not the same in the consecutive transmission time 
slots. Frequency diversity is another technique where a symbol is transmitted by using two or more different frequency bands hoping that all the transmission frequencies don't experience the same fading. Polarization diversity is transmitting a given symbol using differently polarized antennas hopping that the different polarizations will not have the same fading. Angle diversity and spatial diversity are another two diversity techniques. Angle diversity is used at 10 Giga hertz frequencies where there is high scattering effect which results in uncorrelated signal reception at different angles. Spatial diversity uses multiple antennas for transmission and reception which is the focus of this thesis.

The utilization of properly designed spatial diversity increases the SNR at the receiver depending on the type of diversity combining method used. Many diversity combing methods exist including selection combing where the branch with the highest received signal is taken and processed. Switched diversity is another diversity combing technique where there is predefined threshold, if that threshold is achieved the branch will be taken as an output. Equal gain combing (EGC) and maximum ratio combing (MRC) are the two another diversity combing method where the first assumes all the received branches have equal gain but different phase and the second weighs each branch by a proportional amplitude and phase to avoid any biasing in phase and amplitude of the received signal. By merely using multiple antenna techniques we can have larger SNR (called diversity gain) at the receiver. Further improvement of the system performance can be achieved by using space time coding techniques. Many space time coding techniques exist but space time block codes and space time trellis codes are the focus of this thesis. Space time block codes have good diversity gain with a simple decoding algorithm but space time trellis codes achieve both coding gain and diversity gain with more complex viterbi decoding algorithm. In space time coding, symbols to be transmitted are distributed across time and space where space represents transmitter antennas and time represents transmission times. In a given transmission time, all the transmitter antennas will simultaneously transmit different symbols.

In space time block coding, binary bits from the channel encoder are modulated and then space time encoded by the space time encoder where it performs some sign change and symbol rearrangement of the input symbols to fit the number of transmitters and transmission periods. Maximum likelihood decoding (MLD) is used at the receiver to process the signal.

In space time trellis code, a joint modulation and encoding is performed at the transmitter. The binary bits from the channel encoder are fed in parallel to the space time trellis encoder where the trellis encoder uses trellis diagram for encoding. At the receiver, the viterbi algorithm is used to determine the distance metric between actually received and hypothetically received symbols.

\section{STATE OF THE ARTS}

Wireless Communication has made a tremendous impact on the lifestyle of a human being. However, the values of wireless communication are not found for free. Many researchers have also already studied the multipath fading which arises due to the non-coherent combination of signals arriving at the receiver antenna [1]. It is proven via different researches that frequency diversity, solves the multipath problem by transmitting the same information on multiple frequency carriers thereby increasing the probability of undistorted information. The separation between the slots (carriers) has to be at least the coherence bandwidth $\left(B_{C}\right)$ of the channel. A shortcoming of the scheme is that multiple transmitters are needed. In practice, frequency diversity is usually achieved by using spread spectrum signals Such diversity techniques are not bandwidth efficient since all the frequencies within the given bandwidth are occupied by the same information. Another solution is time diversity, the same information is transmitted in different time slots. But the time diversity significantly reduces the throughput of the system. Thus, in practice, diversity is often introduced by adding error correction coding and interleaving [2]. One of the drawbacks of the scheme is that due to the redundancy introduced in the time domain, there is a loss in bandwidth efficiency. Another drawback of this diversity is that it has no effect if it is applied in stationary systems [3]. Many researches have been carried out on both space time trellis codes and space time block codes by using different parameters resulting in possibly different performances.

Space time block codes based on orthogonal designs with full rate and with less than one rate has implemented for different spectral efficiencies using 8-PSK and 16-QAM modulations and the BER has significantly reduced by increasing the number of transmit and receive antennas [4]. An extended closed loop space time block codes has also discussed combined with antenna selection diversity combining method at the receiver and the performance was improved compared to that of the ordinary Qausi-Orthogonal Space time block codes [5]. Unfortunately, such system requires a very complex decoding algorithm at the receiver

Another research was also conducted on super-orthogonal space-time block code using unitary expansion which increases the code rate $(\mathrm{R})$ of the code matrix by using unitary expansion. This results in an increased rate and better SNR performance compared to the orthogonal space time block codes [6]. MIMO 
space-time block coding (STBC) has investigated by considering real and complex signal constellations of space time block codes using M-array QAM and QPSK modulations with different code rates, different transmit and receive antenna configurations and it concluded that low constellation order with high code rate is preferable than high constellation order with low rate [7] and [8]. Furthermore, the research was able to prove that higher diversity gain does not always mean better performance at different SNR.

The performance analysis of space-time trellis codes has investigated to assess the performance of space time trellis codes for different number of sates and M-array PSK modulations [9]. This has shown that for a given modulation scheme, higher number of states $(4,8,16)$ outperforms the lower states. Hamid Jafarkhani et al worked on super-orthogonal space-time trellis and the simulation results has shown that for the same modulation scheme, better SNR performances are found with increasing number of states [10]. The performance of diversity combining has shown great importance of MIMO [11]. The choice of antenna array for beamforming and hence MIMO systems has also attracted researchers [12, 13]. Furthermore, several researches have been conducted on MIMO signal processing and massive MIMO transmission on different cell structures [14-18]. However, they didn't explain the best technique including space coding, number of antennae, modulation type and combining technique that results in to the best bit error performance at minimum cost. Quit recently, a broadband channel estimation algorithm for millimeter wave MIMO systems has developed [19] which uses the joint sparsity of the millimeter wave MIMO channel in the angle and delay domains. However, it doesn't exploit the significance of the time space coding. [20] and [21] have proposed user-scheduling and beamforming method for massive MIMO in which they use a set of orthogonal reference beams and construct a double cone around each reference beam to select semi-orthogonal users based only on channel quality indicator (CQI) feedback. However, it is not optimal (i.e. it costs more) especially for limited number of users.

In general, all of the researches have shown that multipath fading highly degrades the received signal where either large power is transmitted or diversity technique is used. Furthermore, the researches didn't exploit the integrated effect of space time coding, modulation and diversity combining techniques. Transmitting large power is costly in addition to creating interfering signals to the nearby users; that's why spatial diversity is used to reduce deep fades. Because of their size; achieving large SNR is not possible on small devices like cell phones (i.e. on mobile communication). Hence, in this paper, reducing the operating SNR requirement of the mobile device is recommended using space time coding on MIMO systems.

\section{RESEARCH METHOD}

\subsection{Transmit Diversity}

MISO consists of two or more transmitters and single receiver. By simply transmitting a symbol $\mathrm{x}(\mathrm{t})$ from $\mathrm{n}$ different transmitters we can have better SNR at the receiver. In fact, at normal circumstances the transmitter antennas have no channel state information (CSI). Besides, transmit diversity is difficult to extract at the receiver because signals mix while travelling across the wireless environment. As per Shannon's channel capacity, the channel capacity of SISO system is calculated as:

$$
\mathrm{C}=\mathrm{B} * \log 2(1+\mathrm{SNR})
$$

Where B is channel bandwidth and SNR is signal to noise ratio of the received signal

For the MISO system, we have $n_{T}$ antennas at transmitter and only one at receiver. Assuming the channel coefficients are equal and normalized, the channel capacity of MSIO system is given as:

$$
\mathrm{C}=\mathrm{B} * \log 2\left(1+n_{T} * S N R\right)
$$

Where $n_{T}$ is represents the number of transmitter antennas.

As it can be observed from Equation (2), the SNR of the transmit diversity is $n_{T}$ times greater.

\subsection{Receive Diversity}

For the SIMO systems, we have $\boldsymbol{n}_{\boldsymbol{R}}$ antennas at receiver and only one at transmitter. If the signals received on these antennas have on average the same amplitude, then they can be added coherently to produce an $\boldsymbol{n}_{\boldsymbol{R}}{ }^{2}$ increase in the signal power. On the other hand, there are $\boldsymbol{n}_{\boldsymbol{R}}$ sets of noise that are added incoherently and result in an $\mathrm{N}$-fold increase in the noise power. Hence, there is an overall increase in the SNR. The mathematical expression given in (3) assumes all the coefficients are equal and normalized.

$$
\mathrm{C}=\mathrm{B} * \log 2\left(1+n_{R} * S N R\right)
$$


The SNR is $\boldsymbol{n}_{\boldsymbol{R}}$ times that of SISO system which rises with increasing the number of receive antennas. Receive diversity is widely used, for example in uplink. Downlink is more difficult to take advantage of, because the transmitted signals are mixed together, and the transmitter does not have instantaneous CSI [3]. On the other hand, it is difficult to put multiple receive antennas to a mobile device, because of size and power consumption constraints. In contrast, transmit diversity decreases the processing power needed by the receiver.

\subsection{Multiple input multiple output antennas Systems (MIMO)}

MIMO is the use of multiple antennas at both the transmitter and receiver sides to improve communication performance. Compared to both MISO and SIMO antenna configurations, combined effect of transmit and receive diversity results in higher SNR gain at the receiver side of the communication system. If we assume all the channel coefficients are equal and normalized and the same signal is transmitted by each antenna, the channel capacity of the MIMO system is given in (4):

$$
\begin{aligned}
& \mathrm{SNR}=n_{T} n_{R} * \mathrm{SNR} \\
& \mathrm{C}=\mathrm{B} * \log 2\left(1+n_{T} n_{R} * \mathrm{SNR}\right)
\end{aligned}
$$

\section{SPACE TIME CODING}

In space time coding, redundancy is introduced in time and space domains. Space time encoded systems achieve transmit diversity and antenna gain over spatially un-coded systems without sacrificing bandwidth.

\subsection{Space Time Block Codes (STBC)}

In STBC, symbols to be transmitted are distributed across time and space so that each symbol is transmitted by each transmitter antenna at different transmission periods. Since the number of transmitter antennas and the transmission time slots are equal Alamouti space time code has full rate. But Alamouti space time code type of coding is bound only to two transmit antennas. STBC are the generalizations of Alamouti space time codes with arbitrary number of transmit and receive antennas.

Generally, space-time block code is defined by $\boldsymbol{n}_{\boldsymbol{T}} \mathbf{x} \mathbf{p}$ transmission matrix S, where $\boldsymbol{n}_{T}$ represents the number of transmit antennas and $\mathbf{p}$ represents the number time periods needed to transmit one block of coded symbols. The ratio between the number of symbols that space-time block encoder takes as its input (k) and the number of space-time coded symbols transmitted from each antenna defines the rate of a space-time block code [22]-[26]. The rate of STBC can be calculated by:

$$
\mathrm{R}=\frac{K}{P}
$$

The spectral efficiency of the space-time block code is given by

$$
\eta=\frac{r_{B}}{B}=\frac{k m}{P} \mathrm{BPS} / \mathrm{Hz}
$$

Where $r_{B}$ bit rate and $\mathrm{B}$ is is the bandwidth

\subsection{Space Time Trellis Codes (STTC)}

Recent merging research directions suggests that the soft-processing of the signals can result in to significant performance enhancement [27-28]. STBC has no coding gain besides they require bandwidth expansion if code rates are less than one. Ofcourse such codes have simple decoding mechanism. STTCs are based on well defined trellis structures and hence can be decoded using soft-decision decoding techniques at the receiver, such as Viterbi decoding. STTC modulations proposed a joint design of coding, modulation, and transmit diversity for flat Rayleigh fading channels. Considering MIMO system with $\mathbf{n}_{\mathbf{T}}$ transmit and $\mathbf{n}_{\mathbf{R}}$ receive antennas, as shown in Figure 1, the transmitted data are encoded by a STTC encoder.

Assuming a slow Rayleigh fading channel where the channel coefficients are constant within one frame length but independently fade from frame to frame of the transmitted symbols.

STTC Encoder: STTC encoder maps binary data to modulation symbols, where the mapping function is described by a trellis diagram. Let us consider STTC coded M-PSK modulation with $\mathbf{n}_{\mathbf{T}} \operatorname{transmit}$ antennas.

STTC Decoders: The decoding procedure is based on the well-known Viterbi algorithm. However, for space-time codes the Viterbi decoder is modified from the conventional convolutional decoder so that the 
branch metric is computed from the complex inputs and the CSI. The Viterbi decoder is then used to calculate the path through the trellis diagram with the lowest accumulated metric.

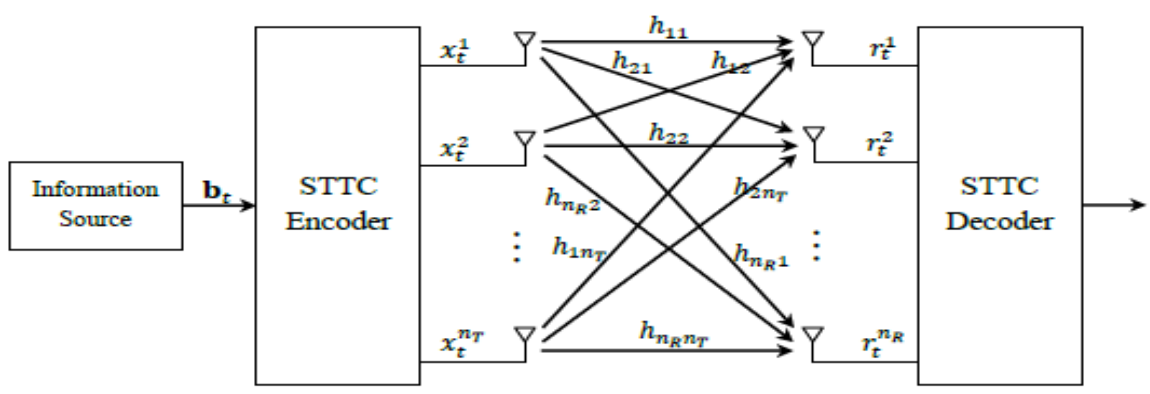

Figure 1. block diagram of STTC coded MIMO system

\section{RESULTS AND ANALYSIS}

The results include the error performance of un-coded diversity system, the SNR performance of the diversity combining techniques, error performance of space time Alamouti codes, error performance of space time trellis codes and space time trellis codes.

In Figure 2, BPSK modulation, Rayleigh fading channel and two transmit antenna and maximum likelihood equalization is used. Accordingly, we can see that as the number of receiver antennas increases, the SNR requirement will significantly fall down. However, simply increasing the number of receiver antennas may not be good ideas since the mobile device size is a big factor in modern mobile communication trends. In addition to this, at higher number of receiver antennas, the SNR reduction is not large as compared to that of when we use smaller number of receiver antennas. Next, we are going to investigate space time coding as a solution to this limitation. The simulation results shown in Figure 3 indicates that MRC is best since it combines many branches of the received signal. Next to the MRC equal gain combining have good SNR gain owing to combining the different branches with unity gain. The selection combining method is simple but has worst performance compared to former two combining methods.

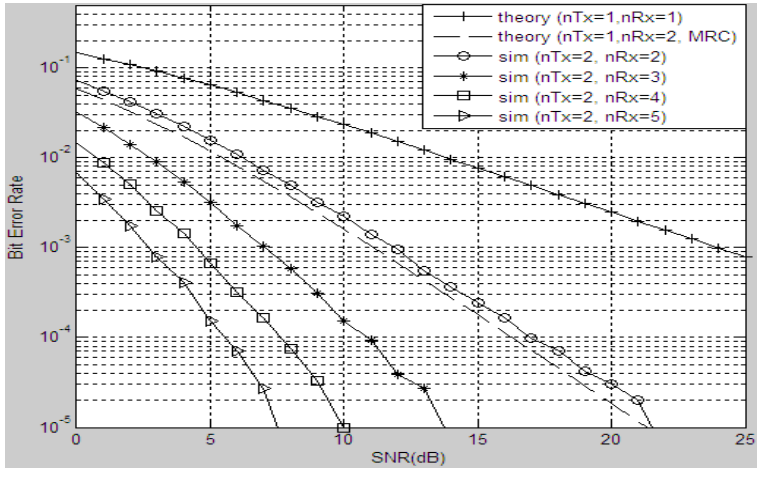

Figure 2: BER performance of un-coded MIMO system for mobile communication

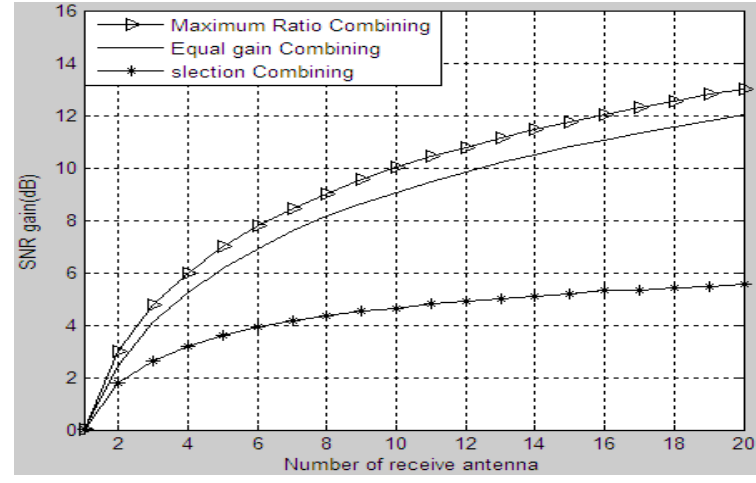

Figure 3: SNR performance of the different diversity combining methods using BPSK

This is because it only uses the branch with the highest power to process the received signal. MRC is more preferable if the number of antennas is greater than four but if the number of antennas is two selection combining can be cost effective. From Figure 4, a loop of $10^{6}$, one transmitter, two receiver antennas are used for each of the diversity combining method. MRC remains the best in terms diversity combing method. Next to MRC, equal gain combing method has a better BER performance compared to selection diversity method. If cost of implementing MRC matters, selection diversity is a best option since the BER incurred by using MRC is not that much large. Conversely, if cost doesn't matter, MRC is the ultimate option. In both Figure 5 and 6, $10^{6}$ symbols are used for each SNR loop and the results indicate that the error probability is reduced if the number of receiver antennas are two as compared to when a single 
antenna is used. The theoretical and simulated results of bit error rate for the Alamouti space time code are approximately the same.

In addition to this, the diversity orders of MRC and Alamouti space time coding are the same as the slopes of the two curves are the same. In contrary to this, the performance of Alamouti code is lower compared to that of MRC because in Alamouti scheme the total power is equally divided to both of the transmitter antennas, where as in MRC, there is a single transmitter antenna which transmits twice that of the Alamouti scheme. The Alamouti scheme can be made equivalent to that of the MRC if both transmitter antennas can transmit the same power as that of the single transmitter MRC. Figure 6 is simulated using 16 PSK modulation, two transmit antennas, and one and two receive antennas. The results have shown that the BER performance is better when the number of receive antennas is two. In fact, the SNR requirement when compared to that of BPSK is large, but this can be compensated by better spectral efficiency of the transmission (i.e. certain bandwidth per number of bits that are transmitted). It is also true that systems with higher modulation order are spectrally efficient but not power efficient. In Figure 7, three transmit and four receive antennas, $10^{5}$ iteration for each SNR and varying M-array PSK modulations are evaluated to observe the BER performance. In this case, even though MIMO is used, the SNR requirement for the higher modulation is bigger. This indicates that there are tradeoffs in higher order M-array scheme which improve spectral efficiency while power requirement to achieve certain error rate rises up significantly. In Figure 7 , there are three transmitter antennas transmitting in four transmission times and hence the operating code rate is $3 / 4$. Though maximum diversity gain advantage is possible in space time block codes, they have no coding gain as those of space time Alamouti codes. In addition to their unity coding gain, non-full rate STBC, especially complex STBC, require bandwidth expansion just like time and frequency diversity. However, such codes have simple decoding mechanism at the receiver. Space time trellis codes are used to achieve both coding gain and diversity gains.

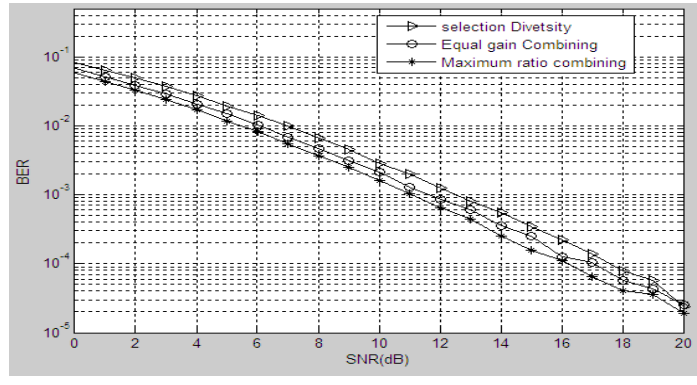

Figure 4. BER performance of different diversity combing methods in Rayleigh fading channel using BPSK

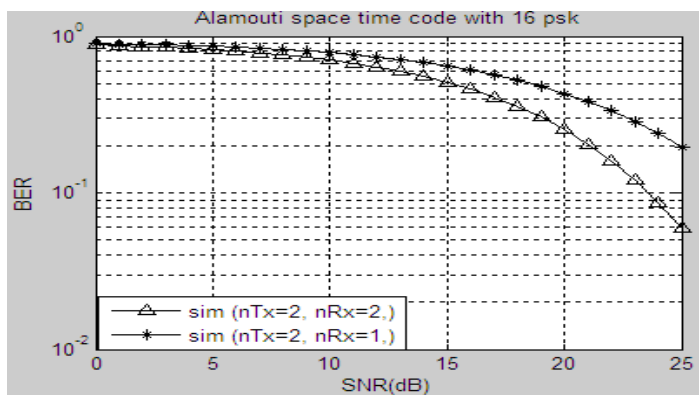

Figure 6. Error performance of 16-PSK modulated Alamouti code

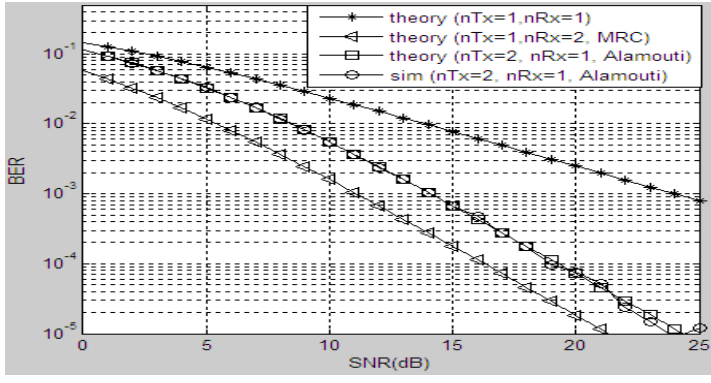

Figure 5. BER performance of BPSK modulated Alamouti code

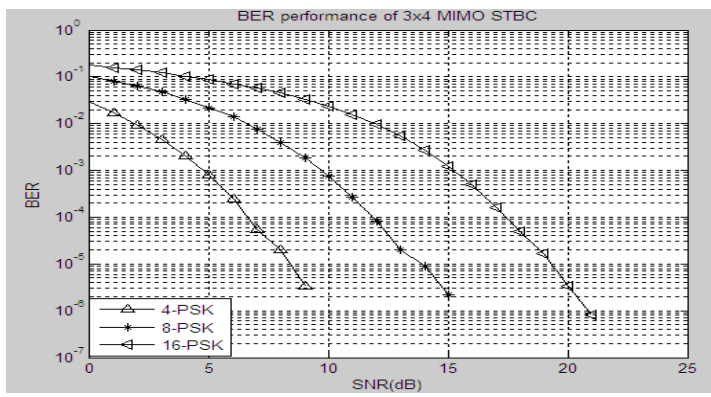

Figure 7. Error performance of M-array PSK modulated $3 \times 4$ space time block codes

Figures 8 and 9 are also numerical plots of error performance using rank and determinant, and trace criteria, respectively. In both cases, the error performance increases with increasing the Eigen values of the transmission matrix. As it can be observed from Figure 10, doubling the number of antennas doesn't necessarily double the error performance. Figure 11 is simulated using different error rates using 4-PSK, 4- 
state 2x2 MIMO configurations, where SER defines symbol error rate, FER defines frame error and PE states pair wise error probability of the system.

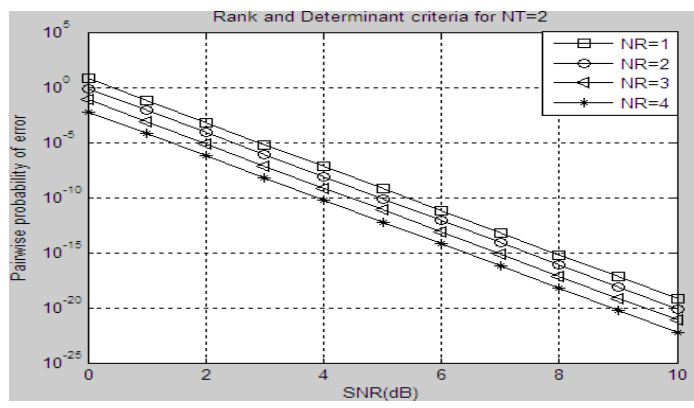

Figure 8. Error performances of space time trellis codes using rank and determinant criteria using two transmit and 1 to 4 receive antennas for high SNR

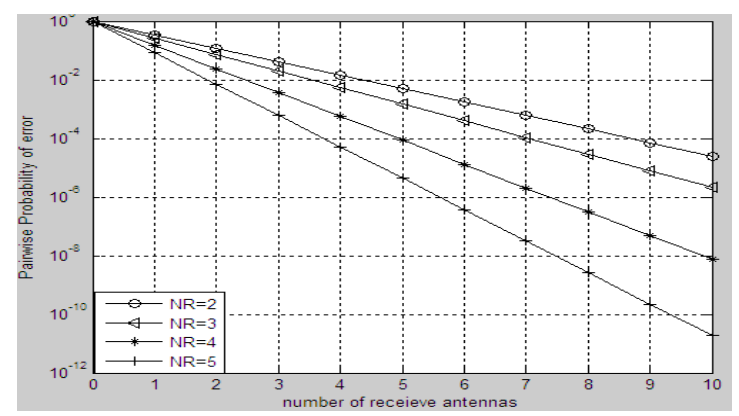

Figure 10. Error Performance of space time trellis codes using different design criteria

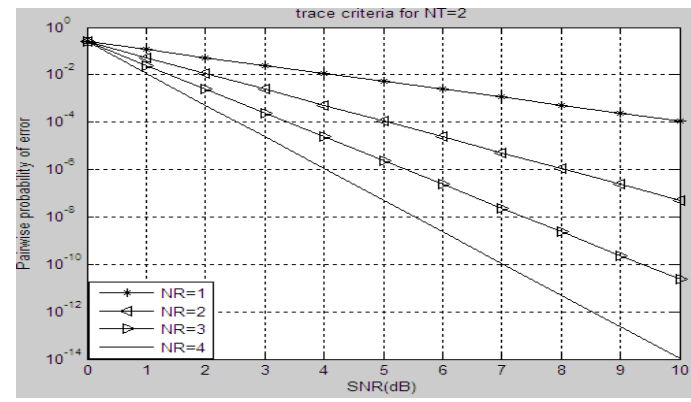

Figure 9. Error Performance of performance of space time trellis codes using trace criteria for two transmit and one to four receive antennas at high SNR

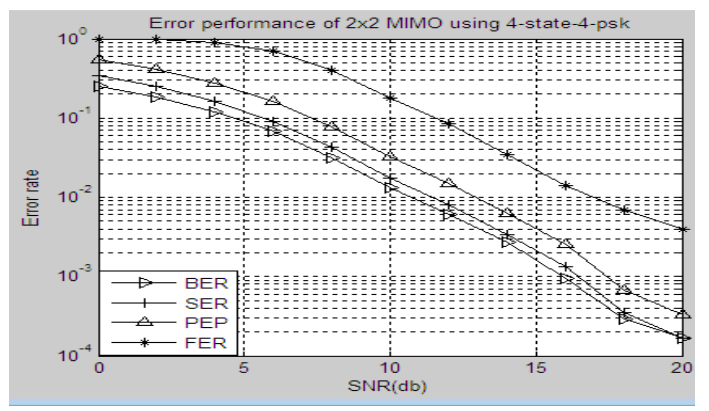

Figure 11. Error Performance of 4-state 4-PSK space time trellis codes for $2 \times 2$ MIMO

Figure 12 indicates that keeping the modulation scheme the same to 4-PSK, varying the number of states with different criteria results in different results of error performance. In the SNR of 0 to 6, the 8-state Trace criteria almost perform the same as that of 32-state rank and determinant criteria. 8 state trace criteria perform better than 16-state trace criteria for all SNR. In addition to this, 32-state has better error performance compared to the 4 -state rank and determinant criteria.

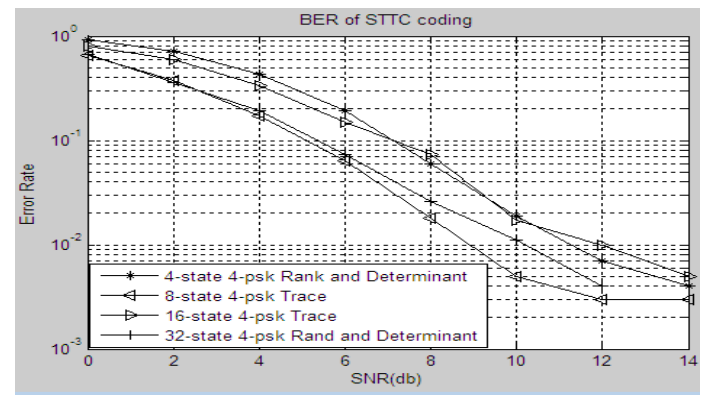

Figure 12. Error Performance of space time trellis codes using trace, rank and determinant criteria

\section{CONCLUSION}

In this work, the error performance of different MIMO systems is studied using different diversity combing methods, space time block codes and space time trellis codes with different parameters including number of antennas, M-array modulations, trace, rank and determinant criteria. The simulation results 
indicate that the performance cannot be doubled by simply doubling the number of antennas. This indicates that there is a limit for the number of antennas at which further increment of antennas cannot result in to significant effect on the system performance. It is also confirmed that STBCs have only diversity gain but no coding gain where as STTCs have both diversities gain and coding gain. If a system with a higher performance is required, STTC is highly recommended. On the other hand, if a system with comparably less performance but cost effective is required, STBC is advisable.

\section{REFERENCES}

[1] Neelam Srivastva, "Diversity schemes for wireless Communication a short Review", Journal of Theoretical and Applied Information Technology, Vol.15, May 31, 2010.

[2] Nirmalendu bikas sinha, R. Bera, and M. Mitra, "Capacity and V-Blast Techniques for MIMO Wireless Channel", Journal of Theoretical and Applied Information Technology, 2005.

[3] Branka Vucetic and Jinhong Yuan, "Space Time Coding”, John Wiley \& Sons Ltd, 2003.

[4] Vahid Tarokh, Hamid Jafarkhani, and A. R. Calderbank, "STBC from Orthogonal Designsm," IEEE transactions on information theory, vol. 45, no. 5, July 1999.

[5] Yi Yu, Sylvie Keroueden, and Jinhong Yuan, "Closed-Loop Extended Orthogonal space time, block codes for three and four transmit antennas," IEEE signal processing letters, vol. 13, No. 5, May 2006.

[6] Heechoon Lee, Massimiliano Sitiy,Weijun Zhu, and Michael P. Fitz, "super-orthogonal space time block code using a unitary expansion," University of California Los Angeles.

[7] Luis Miguel Cort'es-Pe na, "MIMO space times block coding (STBC) simulations and results, personal \& mobile communications," April 2009.

[8] Vinay Dawar and Ritu Sharma, "Reduction in Bit Error Rate from Various Equalization Techniques for MIMO Technology," International Journal of Soft Computing and engineering, Volume-2, September 2012.

[9] Nicky Yuen, "performance analysis of space-time trellis codes," The University of British Columbia, April 2003.

[10] Hamid Jafarkhani, and Nambi Seshadri, "super-orthogonal space-time trellis codes, IEEE transactions on information theory," vol. 49, No. 4, April 2003.

[11] Suvarna P.Jadhav,Vaibhav, and S. Hendre, "Performance of MRC (MRC)," International Journal of Scientific and Research Publications, Volume 3, Issue 2, February 2013.

[12] Lema, Gebrehiwet Gebrekrstos, et al "A novel elliptical-cylindrical antenna array for radar applications." IEEE Transactions on Antennas and Propagation 64.5 (2016): 1681-1688.

[13] Lema, Gebrehiwet Gebrekrstos., et al "Performance Evaluation of Elliptical-cylindrical Antenna Array using SaDE Optimized Hyper Beam." Indonesian Journal of Electrical Engineering and Computer Science 7.1 (2017): 178-188.

[14] Arik, Sercan O., Joseph M. Kahn, and Keang-Po Ho. "MIMO signal processing for mode-division multiplexing: An overview of channel models and signal processing architectures." IEEE Signal Processing Magazine 31.2 (2014): 25-34.

[15] Xie, Hongxiang, et al. "A Unified Transmission Strategy for TDD/FDD Massive MIMO Systems With Spatial Basis Expansion Model." IEEE Trans. Vehicular Technology 66.4 (2017): 3170-3184.

[16] Ngo, Hien Quoc, et al. "Cell-free massive MIMO versus small cells." IEEE Transactions on Wireless Communications 16.3 (2017): 1834-1850.

[17] Pan, Kyle Jung-Lin. "Method and apparatus for efficient precoding information validation for MIMO communications." U.S. Patent No. 9,716,604. 25 Jul. 2017.

[18] Boshkovska, Elena, et al. "Robust resource allocation for MIMO wireless powered communication networks based on a non-linear EH model." IEEE Transactions on Communications65.5 (2017): 1984-1999.

[19] Mo, Jianhua, Philip Schniter, and Robert W. Heath. "Channel estimation in broadband millimeter wave MIMO systems with few-bit ADCs." IEEE Transactions on Signal Processing 66.5 (2018): 1141-1154.

[20] Lee, Gilwon, et al. "A new approach to user scheduling in massive multi-user MIMO broadcast channels." IEEE Transactions on Communications 66.4 (2018): 1481-1495.

[21] Raghavan, Vasanthan, et al. "Millimeter-wave MIMO prototype: Measurements and experimental results." IEEE Communications Magazine 56.1 (2018): 202-209.

[22] Sarita Boolchandani, Garima Mathur, and Gurpriksha Kalra, "Space Time Block Codes: An Overview," International journal of Advances in Computer Networks and its Security.

[23] V. Tarokh, et al, "Space-Time Codes for Wireless Communication: Code Construction," IEEE 47th Vehicular Technology Conference, vol. 2, Phoenix, Arizona, 4-7 May 1997.

[24] V. Tarokh, N. Seshadri, and A.R. Calderbank, "Space-Time Codes for High Data Rate Wireless Communications: Performance Criterion and Code Construction," IEEE Transactions on Information Theory, vol. 44, No. 2, March 1998.

[25] Z. Chen, J. Yuan, and B. Vucetic, “An Improved Space-Time Trellis Coded Modulation Scheme on Slow Rayleigh Fading channels,” IEEE International Conference on Communications, ICC 2001. Vol. 4, June 2001.

[26] Jaspreet singh kaleka, "weighted multilevel space-time trellis codes for Rayleigh fading channels,"2012.

[27] Hailu DH, Gebrehaweria BG, Kebede SH, Lema GG, Tesfamariam GT. Mobile fronthaul transport options in CRAN and emerging research directions: A comprehensive study. Optical Switching and Networking. 2018 Jun 19.

[28] Lema, Gebrehiwet Gebrekrstos, and Dawit Hadush Hailu. "Feasibility study of antenna synthesis using hyper beamforming." Heliyon 5.2 (2019): e01230. 